\title{
Construção de protocolo para elaboração de extrato de Cucumis anguria $L$.
}

\author{
Construction of protocol for the elaboration of Extract of Cucumis anguria L.
}

Construcción de protocolo para la elaboración de Extracto de Cucumis anguria L.

Renan Trindade Pacheco da Silva ORCID: https://orcid.org/0000-0002-8329-4205 Universidade do Estado de Mato Grosso, Brasil E-mail: renan.trindade.pds@gmail.com Cristiane Ferreira Lopes de Araújo ORCID: https://orcid.org/0000-0002-4136-5271 Universidade do Estado de Mato Grosso, Brasil E-mail: cristiane.araujo@unemat.br

Viviane Karolina Vivi-Oliveira ORCID: https://orcid.org/0000-0002-0639-7171 Universidade Federal do Mato Grosso, Brasil E-mail: karolinavivi@gmail.com

Vagner Ferreira do Nascimento ORCID: https://orcid.org/0000-0002-3355-163X Universidade do Estado de Mato Grosso, Brasil E-mail: vagnernascimento@unemat.br

\begin{abstract}
Resumo
Este estudo objetivou construir protocolo de elaboração do extrato hidroetanólico do Cucumis anguria L. Trata-se de estudo do tipo metodológico, a partir de ensaios laboratoriais para obtenção de extrato. Foi testado o método por tintura-mãe hidroetanólico, por maceração, de acordo com a Farmacopeia. Outrossim, foi testado o método de hidrodestilação para extração de óleo essencial. Para a produção do extrato hidroetanólico, por tintura-mãe, os frutos in natura foram adicionados no frasco de vidro contendo o líquido extrator álcool $70^{\circ} \mathrm{GL}$. $\mathrm{O}$ frasco ficou protegido de qualquer alteração do meio externo, sendo agitado diariamente, por 30 dias até ser levado ao evaporador rotativo. O líquido extrator foi eliminado na sua proporção de $70 \%$ da graduação inicial, restando o extrato final, com características de um líquido viscoso. Já para a extração de óleo essencial, por hidrodestilação, foram utilizados o fruto inteiro in natura e as sementes. Após serem inseridos no balonete, aguardou o tempo de fervura, iniciando o cronômetro de 4 horas. Ao final do tempo, não se pode observar a presença de moléculas de óleo suspensas, o que indica que não possuem óleo suficiente para este tipo de método. Na Farmacopeia não possui nenhum teste experimental ou método de extração deste fruto, o que demostra a importância da confecção de um protocolo. Portanto, identificou-se que a extração do Cucumis anguria L. pelo método de tintura-mãe, hidroetanólico, por maceração, com o líquido extrator álcool $70^{\circ} \mathrm{GL}$, demonstrou ser viável para uso experimental.
\end{abstract}

Palavras-chave: Plantas medicinais; Extratos vegetais; Bioprospecção; Cucumis.

\begin{abstract}
This study aimed to build a protocol for the elaboration of the hydroethanolic extract of Cucumis anguria L. This is a methodological study, based on laboratory tests to obtain extracts. The method was tested by hydrothanbolic mother tincture, by macerated, according to the Pharmacopoeia. Moreover, the hydrodistillation method for essential oil extraction was tested. For the production of hydroethanolic extract, by mother tincture, fresh fruits were added to the glass jar containing the liquid extractor alcohol $70^{\circ} \mathrm{GL}$. The vial was protected from any alteration of the external medium, being stirred daily, for 30 days until it was taken to the rotary evaporator. The extractor liquid was eliminated in its proportion of $70 \%$ of the initial graduation, leaving the final extract, with characteristics of a viscous liquid. For the extraction of essential oil, by hydrodistillation, the whole fruit in natura and the seeds were used. After being inserted into the balone, waited for the boiling time, starting the timer of 4 hours. At the end of time, the presence of suspended oil molecules cannot be observed, which indicates that they do not have enough oil for this type of method. In the Pharmacopoeia there is no experimental test or method of extraction of this fruit, which shows the importance of making a protocol. Therefore, it was identified that the extraction of Cucumis anguria L. by the method of mother tincture, hydroethanolic, by maceration, with the liquid extractor alcohol $70^{\circ} \mathrm{GL}$, proved to be viable for experimental use.
\end{abstract}

Keywords: Medicinal plants; Plant extracts; Bioprospecting; Cucumis. 


\begin{abstract}
Resumen
Este estudio tuvo como objetivo construir un protocolo para la elaboración del extracto hidroetanolico de Cucumis anguria $L$. Se trata de un estudio metodológico, basado en pruebas de laboratorio para la obtención de extractos. El método fue probado por tintura madre hidrotanbólica, por macerado, según la Farmacopea. Además, se probó el método de hidrodestilación para la extracción de aceites esencial. Para la producción de extracto hidroetanolico, por tintura madre, se añadieron frutas frescas al frasco de vidrio que contenía el alcohol extractor líquido $70^{\circ} \mathrm{GL}$. El vial fue protegido de cualquier alteración del medio externo, siendo agitado diariamente, durante 30 días hasta que fue llevado al evaporador rotatorio. El líquido extractor fue eliminado en su proporción del 70\% de la graduación inicial, dejando el extracto final, con características de un líquido viscoso. Para la extracción de aceite esencial, por hidrodestilación, se utilizó el fruto entero in natura y las semillas. Después de ser insertado en el balone, esperó el tiempo de ebullición, comenzando el temporizador de 4 horas. Al final de los tiempos, no se puede observar la presencia de moléculas de aceite suspendidas, lo que indica que no tienen suficiente aceite para este tipo de método. En la Farmacopea no existe ninguna prueba experimental o método de extracción de esta fruta, lo que demuestra la importancia de hacer un protocolo. Por lo tanto, se identificó que la extracción de Cucumis anguria L. por el método de tintura madre, hidroetanolica, por maceración, con el alcohol extractor $70^{\circ} \mathrm{GL}$, demostró ser viable para uso experimental.
\end{abstract}

Palabras clave: Plantas medicinales; Extractos de plantas; Bioprospección; Cucumis.

\title{
1. Introdução
}

O Cucumis anguria L. conhecido popularmente no Brasil como maxixe, pertencente à família das Cucurbitáceas. Foi exportado da África para o Brasil durante o período colonial através do tráfico negreiro, do qual este fruto ou também chamado de hortaliça, fazia parte da base culinária dos escravos. Atualmente, pode ser encontrado em qualquer região do Brasil, sendo seu consumo prevalente nas regiões norte e nordeste, onde apresenta duas variantes: o maxixe do Norte (espinhoso) e o maxixe japonês (liso) (Reis, et al., 2019; Silveira, et al., 2015; Silva, et al., 2017).

Pela etnobotânica, o consumo desse fruto está ligado a cicatrização de feridas tegumentares e perda de peso. A grande quantidade de fósforo presente no fruto possui participação na metabolização dos carboidratos via intracelular, sendo comumente consumido por diabéticos, a fim de reduzir os níveis de glicose circulantes na corrente sanguínea (Sousa, et al., 2015; Silva, et al., 2017).

Todavia, ainda que haja tais indicativos de ações terapêuticas, na literatura não há pesquisas para a obtenção do extrato do Cucumis anguria L. para testes experimentais e possível identificação de seus princípios ativos. Além disso, também não se conhece orientações para o melhor método de extração do fruto, porém para frutos em estado in natura, ou seja, recém colhidos, ricos em líquido, indica-se a extração por tintura-mãe (TM). Neste método, ocorre a maceração, processo em que o composto vegetal é extraído dentro de um frasco de vidro fechado, em temperatura ambiente, colocado em contato com o líquido extrator. Comumente, o álcool é o líquido extrator mais utilizado e o prazo da maceração varia, por um período de no mínimo 15 dias, sob agitação ocasional pelo menos uma vez ao dia, conforme é recomendado pela Farmacopeia Homeopática Brasileira (Anvisa, 2011; Sales, 2013; Rodrigues, et al., 2016).

Ao entender que o Cucumis anguria L. pode deixar de ser somente item da alimentação do brasileiro e passar a integrar o acervo da fitoterapia brasileira, que faz parte do Sistema Único de Saúde (SUS) pela Política Nacional de Plantas Medicinais e Fitoterápicos (PNPMF) e pela Política Nacional de Práticas Integrativas e Complementares no SUS (PNPIC), este estudo objetivou construir protocolo de elaboração do extrato hidroetanólico do Cucumis anguria L.

\section{Metodologia}

Trata-se de estudo do tipo metodológico, a partir de ensaios laboratoriais para obtenção de extrato de matéria-prima vegetal (Lima, 2011). O método testado para a preparação foi por tintura-mãe, por maceração, de acordo com as etapas solicitadas pela Farmacopeia Homeopática Brasileira (FHB), em sua última edição (Anvisa, 2011). Os extratos foram produzidos em um laboratório de química, localizado no Centro de Pesquisa, Estudos e Desenvolvimento Agro-Ambientais 
(CPEDA), da Universidade do Estado de Mato Grosso (UNEMAT), do Campus de Tangará da Serra - MT.

Os frutos oriundos da pesquisa foram obtidos através de agricultores que contemplavam os requisitos de não ter utilizado agrotóxicos no maxixeiro e/ou ter utilizado adubo industrial. Os frutos utilizados para o experimento, da espécie Cucumis anguria L. da variante espinhosa, foram coletados na segunda quinzena do mês de maio de 2021, na zona rural do município de Tangará da Serra, em Mato Grosso, Brasil (latitude: $14^{\circ} 37^{\prime} 10^{\prime \prime} \mathrm{S}$; longitude: $57^{\circ} 29^{\prime} 09^{\prime \prime} \mathrm{W}$ e altitude: $387 \mathrm{~m}$ ). Sendo esta época do ano, a transição do período chuvoso para a estiagem (Reis, et al., 2019).

Como solicita a FHB, as espécies de origem vegetal a serem utilizadas em experimentos devem ser coletadas em épocas e em condições adequadas para cada tipo de planta, para um bom resultado da pesquisa, obedecendo a janela de semeadura e colheita, conforme determina a Embrapa (Anvisa, 2011; Maciel, et al., 2017).

A colheita do fruto in natura ocorreu 30 minutos antes de iniciar o processo de fabricação do extrato, tempo este gasto do momento de traslado do fruto da zona rural até o laboratório. Os frutos foram higienizados com água corrente e secados no laboratório de forma natural, sem a utilização de agentes de limpeza, a fim de manter as propriedades químicas e evitar a oxidação da casca do fruto (Costa, 2014; Maciel, et al., 2017).

Após adição dos frutos no recipiente junto com o líquido extrator (solvente), o frasco ficou armazenado em compartimento no laboratório, livre da ação direta de luz, calor e umidade. A tintura-mãe era agitada pelo próprio pesquisador, em movimentos circulares com duração de 10 segundos, uma vez ao dia às 14h00 (horário de Brasília) (Anvisa, 2011).

Para a tentativa de extrair Óleo Essencial (OE), foi utilizado a metodologia de Sartor (2009), por hidrodestilação, utilizando o aparelho Clevenger. Os frutos e as sementes in natura foram higienizados com água corrente e pesados com a balança analítica em $100 \mathrm{~g}$, antes de ser adicionados ao balonete.

Foram respeitados todos os aspectos éticos em pesquisa, tendo início somente após aprovação do Comitê de Ética da Universidade do Estado de Mato Grosso (CEP/ UNEMAT), CAAE: 26137519.1.0000.5166 e com parecer favorável $\mathrm{n}$. 3.779 .749 .

\section{Resultados}

Para a produção do extrato hidroetanólico foi utilizado o método de TM, que é obtido a partir do fruto in natura, preparado exclusivamente por maceração. Os frutos in natura foram previamente higienizados em água corrente e fatiados no eixo horizontal, adicionados em um frasco de vidro contendo o líquido extrator álcool $70^{\circ} \mathrm{GL}$ (Figura 1). O líquido extrator não pode ter concentrações hidroalcóolicas inferiores a 20\%, devido à proliferação microbiana dentro da matéria orgânica (Anvisa, 2011; Sales, 2013).

Figura 1. Frutos acondicionados no frasco e protegido da luz.

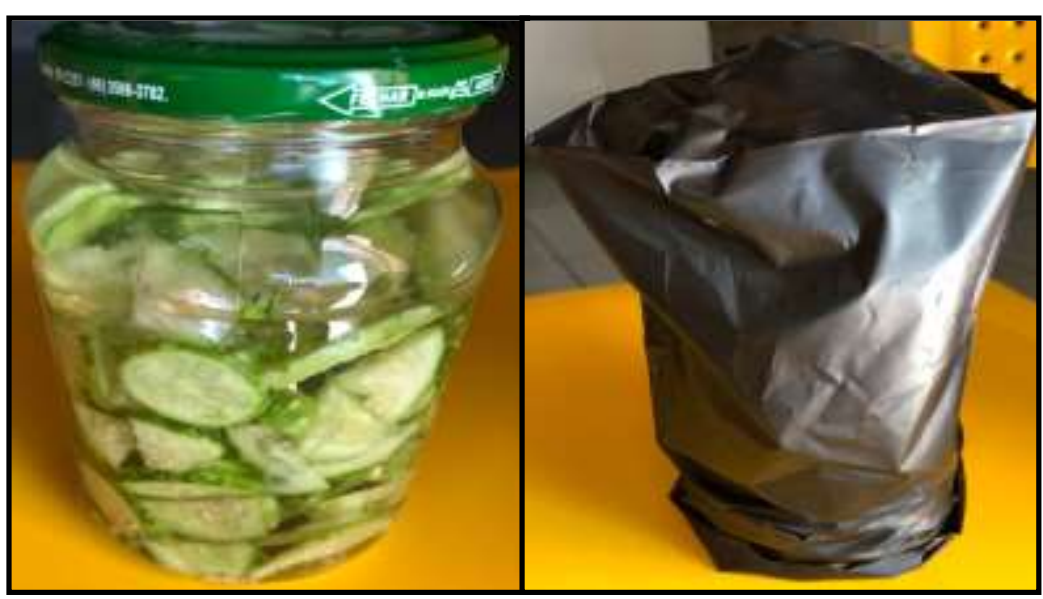

Fonte: Autores. 
Os frutos foram pesados e inseridos no frasco de vidro na proporção de (1:3) em relação ao líquido extrator, armazenados à temperatura ambiente que variou de $20^{\circ} \mathrm{C}$ a $36^{\circ} \mathrm{C}$, durante 30 dias, sem a renovação do líquido extrator e protegido da luminosidade (Figura 1). O frasco ficou protegido de qualquer alteração do meio externo, sendo agitado o recipiente diariamente até sua abertura (Anvisa, 2011).

Após os 30 dias de maceração, o frasco foi descoberto e o líquido separado do material orgânico, através de filtragem com papel filtro. Na sequência, colocou-se em frasco de erlenmeyer, até ser levado para evaporador rotativo. O filtrado da espécie Cucumis anguria L., da variante do Norte (espinhoso), apresentou características organolépticas de coloração verde escuro e aroma adocicado (Figura 2).

Figura 2. Filtrado do Cucumis anguria L. após 30 dias de maceração.

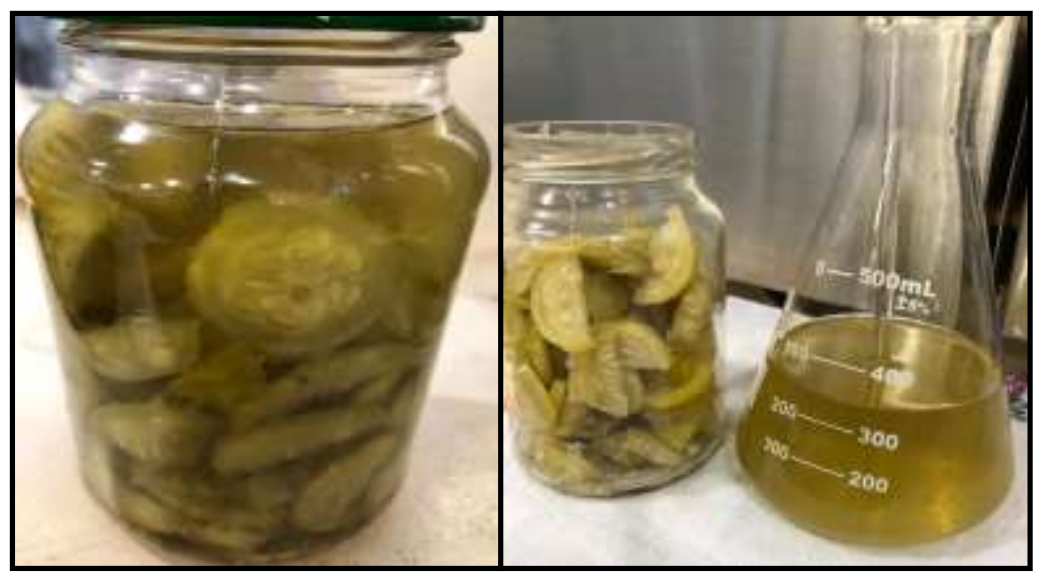

Fonte: Autores.

Após o processo de filtragem e graduação do volume do líquido, o filtrado foi levado ao evaporador rotativo sob uma pressão constante de $600 \mathrm{mmHg}$, interligado ao refrigerador de recirculação em circuito fechado, durante 6 horas, em uma temperatura entre $78,37^{\circ} \mathrm{C}$ a $90,0^{\circ} \mathrm{C}$ (temperatura de ebulição do álcool), sem ultrapassar $>96,0^{\circ} \mathrm{C}$ (temperatura próxima da ebulição da água) (Figura 3).

Figura 3. Operação do evaporador rotativo na temperatura de $80.1^{\circ} \mathrm{C}$.

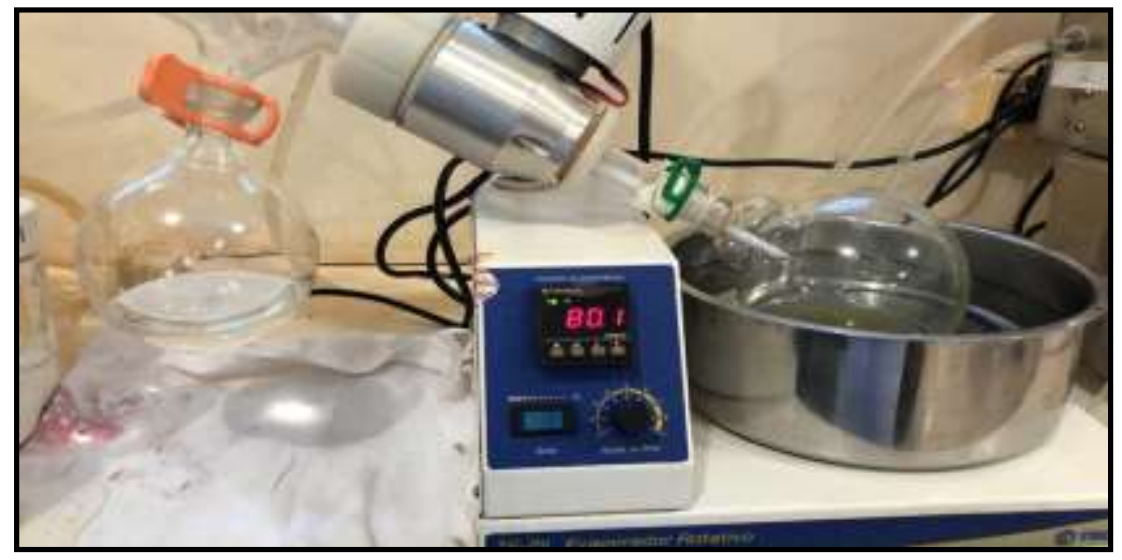

Fonte: Autores.

O líquido extrator foi eliminado na sua proporção de $70 \%$ da graduação total inicialmente, restando o extrato no frasco de evaporação (30\%), que apresentou características de um líquido viscoso. O extrato final da TM obtida foi envasado 
em frasco de vidro âmbar 200ml, armazenado e conservado em geladeira vertical frost free a $4 \pm 1^{\circ} \mathrm{C}$.

Outrossim, foi testado o método de hidrodestilação para a tentativa de extração de (OE), em aparelho Clevenger, durante 4 horas depois do início da fervura (Sartor, 2009). As tentativas de extrações de OE foram realizadas utilizando duas partes, o fruto inteiro in natura da variante espinhosa e o outro teste que utilizou somente as sementes, batidas em liquidificador de jarra de vidro (Figura 4).

Figura 4. Preparo dos frutos e sementes da variante espinhosa, para tentativa de obter OE.

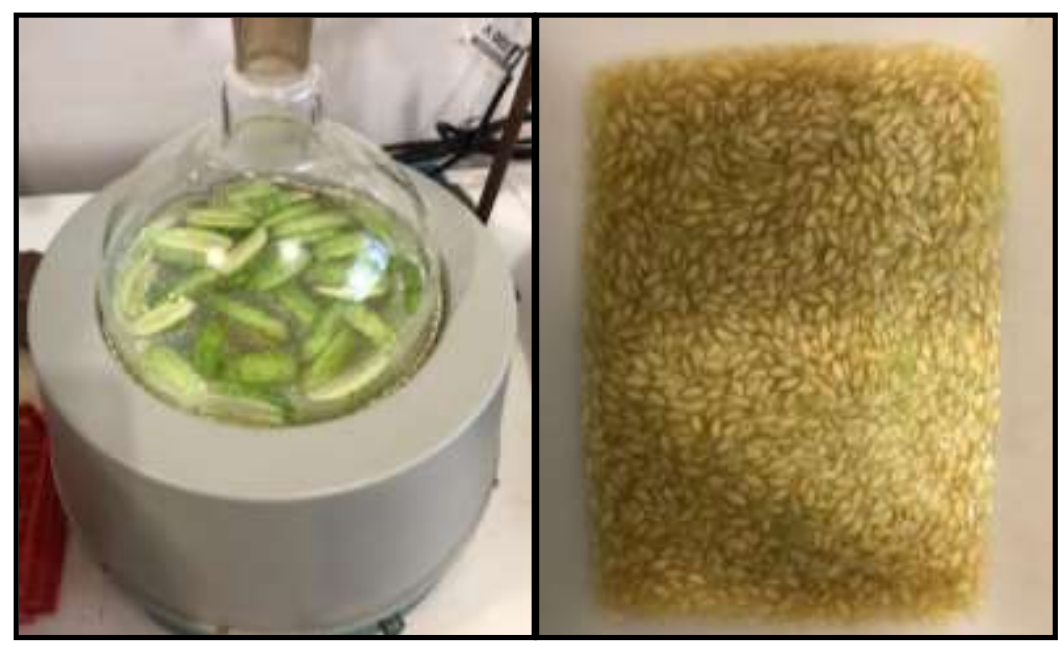

Fonte: Autores.

Para o processo de hidrodestilação do fruto e sementes, realizou os ensaios em triplicada, os frutos foram higienizados em água corrente, cortados no eixo vertical e pesados. Após serem inseridos no balonete do Clevenger, aguardou o tempo de fervura, iniciando assim o cronômetro com duração de 4 horas, como mostra a (Figura 5) momento da fervura do fruto e sementes.

Figura 5. Processo de hidrodestilação do fruto e sementes da variante espinhosa.

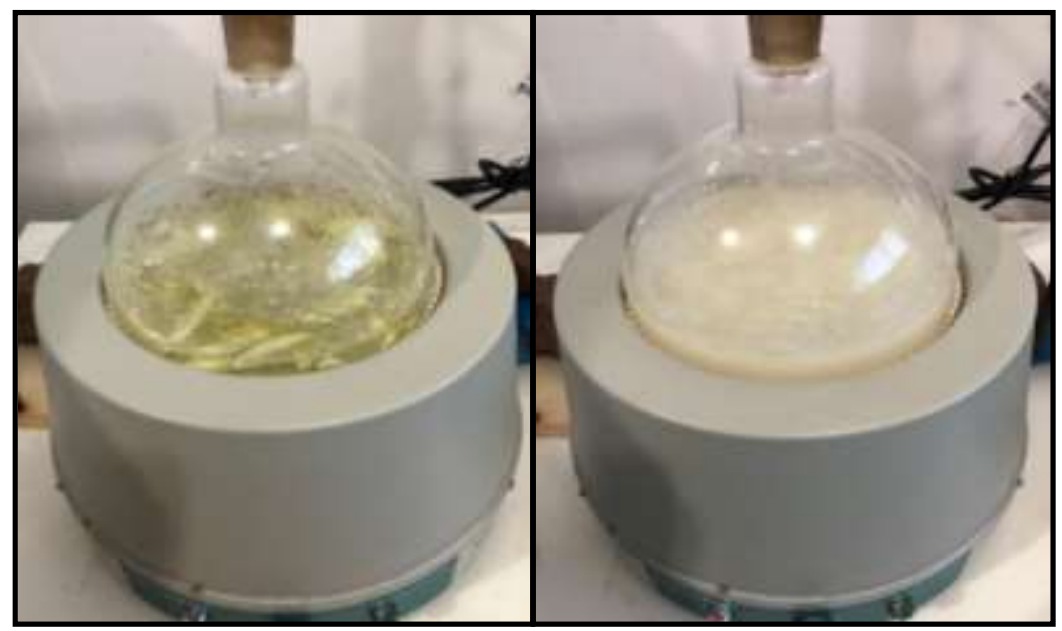

Fonte: Autores.

Ao final do tempo, não se pode observar a presença de moléculas de óleo suspensas, o que indica que o fruto e as sementes em estado in natura, da espécie espinhosa, não possui óleo suficiente para o método de hidrodestilação. 


\section{Discussão}

Na FHB (2011) não possui nenhum teste experimental e/ou as características organolépticas da TM utilizando o Cucumis anguria L., bem como nenhum outro método de extração deste fruto, o que demostra a importância da confecção de um protocolo para elaboração do extrato. A planta mais próxima estudada que FHB traz, pertence à família das Cucurbitáceas, da espécie chamada Bryonia alba L., onde foi utilizado sua raiz para realizar a TM, e o filtrado resultante da maceração ficou com características de cor amarelo-claro, odor nauseoso, desagradável e de sabor amargo FHB (2011), onde apresentou características organolépticas semelhantes à do filtrado do Cucumis anguria L.

Sales (2013) foi o pioneiro em realizar o protocolo para elaboração da TM, por maceração, com os frutos e folhas da aroeira (Schinus terebinthifolius raddi) seguindo os mesmos procedimentos utilizados no presente estudo. Além disso, o autor evidenciou que este método de extração garante a estabilidade do produto, e que proporcionou resultados promissores na determinação do perfil cromatográfico das amostras analisadas (Sales, 2013).

A metodologia utilizada por Oliveira (2017), seguiu o processo de TM semelhante com o presente estudo, utilizando flores de arnica (Solidago chilensis), submetidos à extração por maceração com álcool $66^{\circ} \mathrm{GL}$. O filtrado ficou com coloração verde, odor e sabor aromático, levemente amargo, com características organolépticas semelhantes ao filtrado do Cucumis anguria L.

O trabalho de Costa (2019) traz a importância do controle de qualidade e segurança ao termino da preparação das TM, utilizando metodologias químicas e físico-químicas para avaliar o extrato final, como a determinação de taninos, flavonoides, dentre outras substâncias. Entretanto, para realizar o presente estudo com o Cucumis anguria L. utilizou-se os equipamentos ao alcance dos laboratórios do CPEDA, o que acabou limitando as análises finais físico-químicas mais robustas.

Sobre a escolha da concentração do líquido extrator (álcool $70^{\circ} \mathrm{GL}$ ), um estudo apresentou os resultados que permitiu sugerir que as soluções hidroalcóolicas a $60 \%$ ou a $70 \%$ foram as mais apropriadas para a extração dos polifenóis de folhas de chá verde (Camellia sinensis), e que soluções baixas desta porcentagem, pode haver problemas de conservação com o extrato e dificuldades de concentração das tinturas (Santos, et al., 2014).

A TM hidroetanólica de frutos frescos tem a validade aproximada de 12 meses, podendo ser utilizada externamente na forma de unguentos, fricções, avaliação antimicrobiana in vitro e na formulação de pomadas e cremes (Eller, et al., 2015). A partir do extrato hidroetanólico da TM é possível realizar análises antimicrobianas, antifúngicas, anti-helmínticas e dentre outras. O estudo de Tuama e Mohammed (2019) evidenciou com o extrato do Cucumis sativus (pepino), da mesma família das Cucurbitáceas, ação antimicrobiana e ação anticancerígena satisfatória.

A extração de compostos vegetais possui vantagens e restrições, as principais vantagens é o fácil acesso para a população e o custo acessível. Em contrapartida, as restrições estão no controle de qualidade com a realização de testes físicoquímicos, instabilidade dos compostos orgânicos presentes nos extratos e as possíveis substâncias tóxicas presentes nas plantas, principalmente quando os extratos são produzidos de forma caseira, sem protocolos, e quando utilizados de forma errônea (Santos, et al., 2014).

Sobre a tentativa de extrair OE, Busato (2014) explica que existe várias condições na hidrodestilação que dificulta a extração de OE, sendo um deles, quando a matéria orgânica (frutos e folhas) utilizadas, possui grande quantidade de água, dificultando a concentração do óleo extraído. A melhor forma de utilizar o método por hidrodestilação é com a matéria orgânica seca. Isso explica porque que não houve a presença de moléculas de óleo suspensas do Cucumis anguria L. em seu estado in natura. Outrossim, sobre o método de hidrodestilação, a literatura encontra-se apenas estudos utilizando as folhas secas, são raros os estudos utilizando o fruto, ainda mais em seu estado in natura, o que acaba inviabilizando este método como extração para o fruto do Cucumis anguria L. (Sales, 2013).

O extrato hidroetanólico do Cucumis anguria L. possui uma alta perspectiva de ação terapêutica e indicativos de 
evidências, pelas constantes indicações populares. Na oportunidade de identificar o potencial terapêutico do extrato do Cucumis anguria L., ele será adicionado na Relação Nacional de Plantas Medicinais do Ministério da Saúde (MS), criado desde 2008 (Brasil, 2009). Isso, a médio ou longo prazo poderá constituir caminho para a formulação de um novo fármaco para uso da população, e trazer benefícios frente a diversos agravos à saúde.

\section{Conclusão}

Nesse estudo, identificou-se que a extração de extrato do Cucumis anguria L. in natura pelo método de tintura-mãe, hidroetanólico, por maceração, utilizando o líquido extrator álcool $70^{\circ} \mathrm{GL}$, durante 30 dias demonstrou ser viável para uso experimental. Para tanto, estudos complementares devem ser realizados, a fim de mapear e caracterizar os grupos de compostos químicos presentes no extrato, como por exemplo, o método de cromatografia e/ou espectroscópico, para validação, padronização, garantia da segurança e eficácia da tintura.

O método de extração por hidrodestilação para obtenção de OE não apresentou eficiência para o fruto e sementes em seu estado in natura. Novos experimentos com o fruto seco podem permitir a obtenção de OE, assim como a possibilidade de avaliar se ocorre diferenças organolépticas ou de nutrientes em relação ao fruto em seu estado in natura, uma vez que, o fruto possui alta taxa de senescência e oxidação após a colheita.

\section{Referências}

ANVISA. (2011). Farmacopeia Homeopática Brasileira (3a ed). ANVISA. http://antigo.anvisa.gov.br/en_US/farmacopeia-homeopatica

Busato, N. V., Silveira, J. C., Costa, A. O. S. \& Junior, E. F. C. (2014). Estratégias de modelagem da extração de óleos essenciais por hidrodestilação e destilação a vapor. Ciência Rural, 44(9), 1574-1582. https://www.scielo.br/j/cr/a/gvHbsFT68gFVPfBksDYYhnL/?lang=pt\&format=pdf

Brasil. (2009). Programa Nacional de Plantas Medicinais e Fitoterápicos. Brasília: Ministério da Saúde. https://bvsms.saude.gov.br/bvs/publica coes/programa_n acional_plantas_medicinais_fitoterapicos.pdf

Costa, H. G. (2014). Produtividade de Cultivares de Maxixe (Cucumis anguria L.) em diferentes sistemas de cultivo em duas épocas. Dissertação de PósGraduação, Universidade Federal do Amazonas, https://tede.ufam.edu.br/bitstream/tede/7068/2/Disserta\%C3 \%A7\%C3\%A3o_HerodilsonCosta_PPGAT.pdf

Costa, L. D. S. (2019). Quantificação de fenólicos totais em tintura de jatobá (Hymenaea spp). Monografia, Universidade Federal de Campina Grande, Cuité, PB, Brasil. http://dspace.sti.ufcg.edu.br:8080/jspui/bitstream/riufcg/12144/1/LEONARDO\%20DOS\%20SANTOS\%20COSTA\%20TCC\%20\%20FARM\%C3\%81CIA\%20\%202019.pdf

Eller, S. C. W. S., Feitosa, V. A., Arruda, T. A., Antunes, R. M. P., \& Catão, R. M. R. (2015). Avaliação antimicrobiana de extratos vegetais e possível interação farmacológica in vitro. Revista de Ciências Farmacêuticas Básica e Aplicada, 36(1), 131-136. https://rcfba.fcfar.unesp.br/index.php/ojs/article/view/76

Lima, D. V. M. (2011). Desenhos de pesquisa: uma contribuição ao autor. Online braz. J. nurs., 10(2). http://www.objnursing.uff.br/index.php/nursing/article/view/3648

Maciel, S. R. (2017). A cultura do maxixe. Emater. http://biblioteca.emater.df.gov.br

Nepa. (2011). Tabela brasileira de composição de alimentos - TACO (4a ed). Campinas: BookEditora. https://www.cfn.org.br/wpcontent/uploads/2017/03/taco_4_edicao_ampliada_e_revisada.pdf

Oliveira, T. B., Bastos, B. P. M., Kelly, A. M., Monteiro, S. S., \& Valverde, S. S. (2017). Caracterização de flavonoides por CLAE-UV-PDA em tintura de inflorescências de Solidago chilensis Meyen cultivada em Itaipava (RJ). Revista Fitos, 11(1), 17-25. https://revistafitos.far.fiocruz.br/index.php/revistafitos/article/view/397

Reis, A., Lima, M. F., Lopes, C. A., \& Pinheiro, J. B. (2015). Principais doenças do maxixeiro no Brasil. Brasília: Embrapa. https://www.infotec a.cnptia.embrapa. br/infoteca/handle/doc/1029246

Rodrigues, F. A., Pimenta, V. S. C., Braga, K. M. S., \& Araújo, E. G. (2016). Obtenção de extratos de plantas do cerrado. Enciclopédia Biosfera, 13(23), 870887. http://www.conhecer.org.br/enciclop/2016a/agrarias/obtencao\%20de\%20extatos.pdf

Sales, M. D. C. (2013). Avaliação e caracterização de insumos bioativos da Aroeira (Schinus terebinthifolius Raddi) com potencial econômico para o desenvolvimento tecnológico de bioprodutos. Tese de Doutorado, Universidade Federal do Espírito Santo, Vitória, ES, Brasil. https://repositorio.ufes.br/bitstream/10/4512/1/tese_6111_Tese_Maria\%20Diana\%20C\%20Sales.pdf

Santos, C. B. et al. (2014). Preparo e caracterização de tinturas das folhas de chá verde (Camellia sinensis (L.) O. Kuntze) Theaceae. Rev. Bras. Pl. Med., 16(4), 826-831. https://www.scielo.br/j/rbpm/a/xTCz34NpgNQ5DThbCyQdBQH/?lang=pt 
Research, Society and Development, v. 10, n. 10, e522101019019, 2021

(CC BY 4.0) | ISSN 2525-3409 | DOI: http://dx.doi.org/10.33448/rsd-v10i10.19019

Sartor, R. B. (2009). Modelagem, simulação e otimização de unidade industrial de extração de óleos essenciais por arraste a vapor. Dissertação de Mestrado, Federal do Rio Grande do Sul, Porto Alegre, RS, Brasil. https://www.lume.ufrgs.br/bitstream/handle/10183/21924/000737903.pdf

Silva, G. A., Marques, L. F., \& Oliveira, C. A. (2017). Caracterização físico-quimica dos frutos do maxixe (Cucumis anguria L). II Congresso Internacional das Ciências Agrárias COINTER - PDVAgro, 1(1), 1-7. https://cointer-pdvagro.com.br/wp-content/uploads/2018/02/CARACTERIZA\%C3\%87\%C3\%83OF\%C3\%8DSICO-QUIMICA-DOS-FRUTOS-DO-MAXIXE-Cucumis-anguria.pdf

Silva, R. T. P., Pereira, T. N. S., Neres, C. R. P., Trettel, A. C. P. T., \& Nascimento, V. F. (2019). Propriedades químicas do Cucumis anguria L. em ações terapêuticas com seres humanos. MT Horticultura, 5(2), 80. https://www.mthorticultura.com.br/revista/volume-5-numero-2/

Silveira, P. T. S., Silva, N. M. C., Reis, M. F. T., Landim, L. B., \& Aquino, A. A. (2015). Qualidade pós-colheita do maxixe (Cucumis anguria L.) revestido com amido de milho adicionado do extrato de própolis. Revista Brasileira de Tecnologia Agroindustrial, 9(2) 1888-1899. https://periodicos.utfpr.edu.br/rbta/article/view/1953

Sousa, A. P. B., Lima, F. G. S., \& Lima, A. (2015). Propriedades nutricionais do maxixe e do quiabo. Rev. Saúde em Foco, 2(1), 113-129. http://www4.unifsa.com.br/revista/index.php/saudeemfoco/article/view/688

Tuama, A. A. \& Mohammed, A. A. (2019). Phytochemical screening and in vitro antibacterial and anticâncer activities of the aqueous extract of Cucumis sativus. Saudi Journal of Biological Sciences, 26(3), 600-604. https://www.sciencedirect.com/science/article/pii/S1319562X18301736 